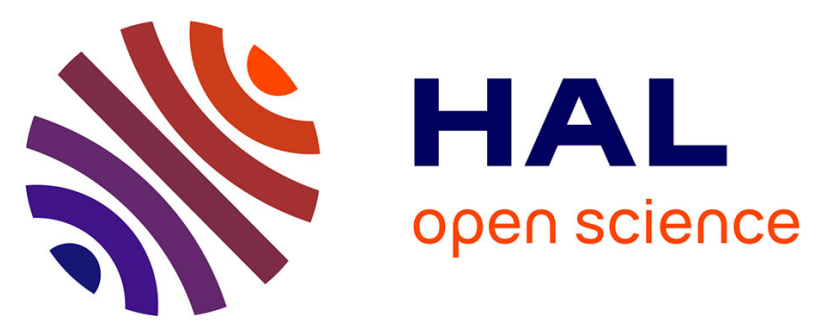

\title{
Ribosomal synthesis and folding of peptide-helical aromatic foldamer hybrids
}

Joseph M Rogers, Sunbum Kwon, Simon J Dawson, Pradeep K Mandal, Hiroaki Suga, Ivan Huc

\section{To cite this version:}

Joseph M Rogers, Sunbum Kwon, Simon J Dawson, Pradeep K Mandal, Hiroaki Suga, et al.. Ribosomal synthesis and folding of peptide-helical aromatic foldamer hybrids. Nature Chemistry, 2018, 10 (4), pp.405-412. 10.1038/s41557-018-0007-x . hal-01849059

\section{HAL Id: hal-01849059 \\ https://hal.science/hal-01849059}

Submitted on 25 Jul 2018

HAL is a multi-disciplinary open access archive for the deposit and dissemination of scientific research documents, whether they are published or not. The documents may come from teaching and research institutions in France or abroad, or from public or private research centers.
L'archive ouverte pluridisciplinaire HAL, est destinée au dépôt et à la diffusion de documents scientifiques de niveau recherche, publiés ou non, émanant des établissements d'enseignement et de recherche français ou étrangers, des laboratoires publics ou privés. 


\section{Ribosomal synthesis and folding of peptide-helical aromatic foldamer hybrids}

Joseph M Rogers, ${ }^{1}$ Sunbum Kwon, ${ }^{2}$ Simon J. Dawson, ${ }^{2}$ Pradeep K. Mandal, ${ }^{2}$ Hiroaki Suga*1 and Ivan Huc $\mathrm{H}^{\star 2 \ddagger}$

1 Department of Chemistry, Graduate School of Science, The University of Tokyo, 7-3-1 Hongo, Bunkyo-ku, Tokyo 113-0033, Japan

2 CBMN Laboratory, Univ. Bordeaux, CNRS, IPB, Institut Européen de Chimie et Biologie, 2 rue Escarpit, 33600 Pessac, France.

* Correspondence to: hsuga@chem.s.u-tokyo.ac.jp, ivan.huc@cup.Imu.de.

‡ Present address: Department of Pharmacy. Ludwig-Maximilians-Universität, Butenandtstr. 5, 81377 München, Germany.

\section{Abstract (N.B. 100-150 words)}

Translation, the mRNA templated synthesis of peptides by the ribosome, can be manipulated to incorporate variants of the twenty cognate amino acids. Such approaches for expanding the range of chemical entities that can be produced by the ribosome may accelerate the discovery of molecules that can perform functions for which poorly folded, short peptidic sequences are ill suited. Here we show that the ribosome tolerates some artificial helical aromatic oligomers, so-called foldamers. Using a flexible tRNA-acylation ribozyme, flexizyme, foldamers were attached to tRNA, and the resulting acylated tRNAs were delivered to the ribosome to initiate the synthesis of noncyclic and cyclic foldamer-peptide hybrid molecules. Passing through the ribosome exit tunnel requires the foldamers to unfold. Yet foldamers encode sufficient folding information to influence the peptide structure once translation is completed. We also show that in cyclic hybrids, the foldamer portion can fold into a helix and force the peptide segment to adopt a constrained and stretched conformation.

\section{(Main Text)}

In peptide biosynthesis, the ribosome, a large ribonucleoprotein complex ${ }^{1}$, progresses along an mRNA template and brings together a succession of adapter tRNAs whose 3'-end hydroxyl groups are acylated with cognate amino acids. Three nucleotides of each tRNA (anticodon) match a given set of three nucleotides on the mRNA (codon) and one amino acid is delivered to the growing peptide chain. The nascent peptide emerges out of the so-called ribosome exit tunnel. Specific aminoacylation of tRNAs, catalysed by the corresponding aminoacyl-tRNA 
synthetases, dictate codons' assignment and constitute the genetic code. Several methods have been developed to artificially expand ${ }^{2-4}$ or reprogram ${ }^{5,6}$ the genetic code by attaching nonproteinogenic amino acids to tRNAs. For example, versatile tRNA-acylation ribozymes, known as flexizymes ${ }^{5}$, conveniently catalyse such attachments. Nonproteinogenic aminoacyltRNAs prepared by flexizymes can be added to solutions containing the translationassociating proteins, cofactors, and ribosomes required for in vitro translation ${ }^{7}$, allowing for the incorporation of a variety of synthetic building blocks ${ }^{5}$ and expanding the chemical register of mRNA-encoded peptides ${ }^{8}$.

Genetic code reprogramming has also revealed the tolerance of the ribosomal machinery to non-proteinogenic amino acids: $\alpha$-amino acids bearing non-natural side chains ${ }^{5}$, many $\mathrm{D}$-amino acids ${ }^{9,10}$ and even some $\beta$-amino acids ${ }^{11}$ have been incorporated within peptidic sequences. Furthermore, chemistry that permits cyclization ${ }^{12}$, as well as ester and $\mathrm{N}$ alkylated amide functions ${ }^{13,14}$ have been shown to be accepted by the ribosome. In addition to single amino acids, preformed di-, tri- or penta-peptides ${ }^{15}$ and other organic building blocks ${ }^{16}$ have also been attached directly to tRNA ${ }^{\mathrm{fMet}} \mathrm{CAU}$, the tRNA that initiates peptide synthesis by matching the start AUG codon of mRNA, and were incorporated as small appendages at the $\mathrm{N}$-terminus of the translated peptide. These developments open up the prospect of hijacking the ribosome machinery in order to produce mRNA encoded chemical entities ever more remote from $\alpha$-peptides, with the notable advantages that (I) mRNA encoding gives access to sequence randomization and selection from libraries containing trillions of unique sequences ${ }^{17}$; and (ii) that enriching chemical diversity increases the range of accessible functions, including resistance to proteolytic degradation, cell penetration capabilities or protein recognition through the folding of short sequences. The stakes are thus

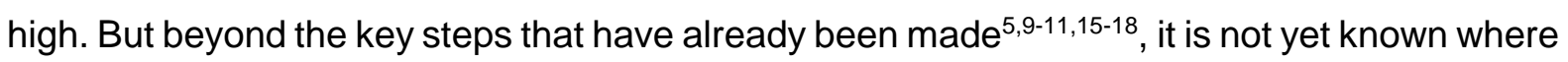
definite limitations lie in this endeavour.

In the following, we present our discovery that the ribosome accepts objects far larger, and more distinct from peptides, than those previously considered. Specifically, we show that helical aromatic foldamers ${ }^{19}$ - helically folded oligomeric backbones having aryl rings in their main chain - larger than $900 \mathrm{Da}$ can be incorporated at the $\mathrm{N}$-terminus of a translated peptide or protein. In the resulting foldamer-peptide hybrids, the peptide component may represent less than half of the whole entity. The process was found to require foldamer unfolding to pass the exit tunnel. Yet, these foldamers' folding propensity largely exceeds that of short peptides and their incorporation amounts to encoding additional folding information that can effectively bias the peptide structure. For example, we show that macrocycles can be translated by the ribosome in which a foldamer segment adopts a helical conformation and forces a peptide segment to remain extended. Reciprocally, the peptide segment controls the handedness of 
the foldamer helix. These results expand the scope of ribosomal expression of mRNA encoded non-natural sequences and also introduce the benefits of using foldamers' stable conformations to control peptide conformation.

\section{Results and Discussion}

Our initial motivation stemmed from the consideration that short peptides are inherently flexible and not prone to folding, whereas some aromatic foldamers feature outstanding conformational stability and might thus serve as structural templates to assist peptide folding. Several approaches already exist to stabilize peptide conformation, including yet not limited to the use of non-natural building blocks such as $\beta$-amino acids ${ }^{20}$ or turn mimics ${ }^{21}$; macrocyclization, e.g. through the stapling of side chains in alpha-helices ${ }^{22}$ or by joining the ends of a sequence; and conformational templates that initiate $\alpha$-helicity ${ }^{23,24}$. Non-natural building blocks and cyclization have also been combined ${ }^{25}$. These conformationally restrained peptides generally perform better in the tight and selective recognition of biomolecules, as opposed to flexible sequences that must pay a higher entropic cost upon binding.

In contrast, some foldamers have superior folding properties to peptides, and even to peptides containing unnatural amino acids ${ }^{26,27}$. Aromatic oligoamide foldamers are in this class: oligomers derived from 8-amino-2-quinolinecarboxylic acid ${ }^{19}$ (henceforth referred to as Q, Fig. 1a) predictably form helical structures with remarkable stability, including in water ${ }^{28,29}$. For example, an octamer shows no denaturation at $120^{\circ} \mathrm{C}$ in $\mathrm{DMSO}^{19}$. $\mathbf{Q}$ monomers are comparable to dipeptides in size (Fig. 1a) but, with a rigid aromatic structure, fewer rotatable bonds and a different pattern of hydrogen bond donors and acceptors, their sequences form distinctly non-proteinogenic helices with a diameter about twice as large as that of an $\alpha$-helix (Fig. 1b). Like peptides, $\mathbf{Q}$ oligomers can be decorated with different side-chains. However, quite unlike peptides, folding is a property of the backbone and is side-chain independent. As a hint that robust folding of $\mathbf{Q}_{n}$ might be conferred to peptides, they have been shown to be stable enough to accommodate individual $\alpha$-amino acids, the conformation of which is then dictated by the aromatic backbone ${ }^{30,31}$. We thus engaged in assessing the extent to which peptide-foldamer hybrid structures could be produced by ribosomal translation of mRNA and possibly exhibit synergistic folding properties. 
a<smiles>CNCC(=O)NCC(C)=O</smiles>

Gly-Gly<smiles>[R]c1cc(C(C)=O)nc2c(NC)cccc12</smiles>

$\mathbf{Q}$

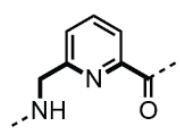

$\mathbf{P}$
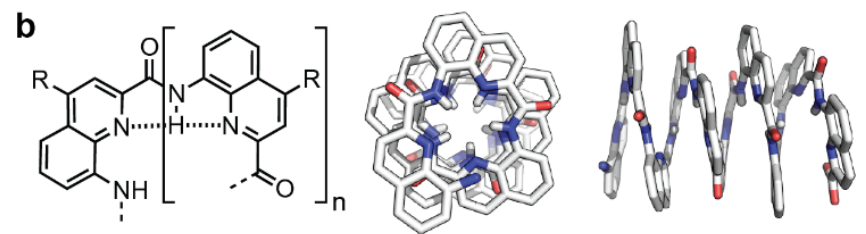

C
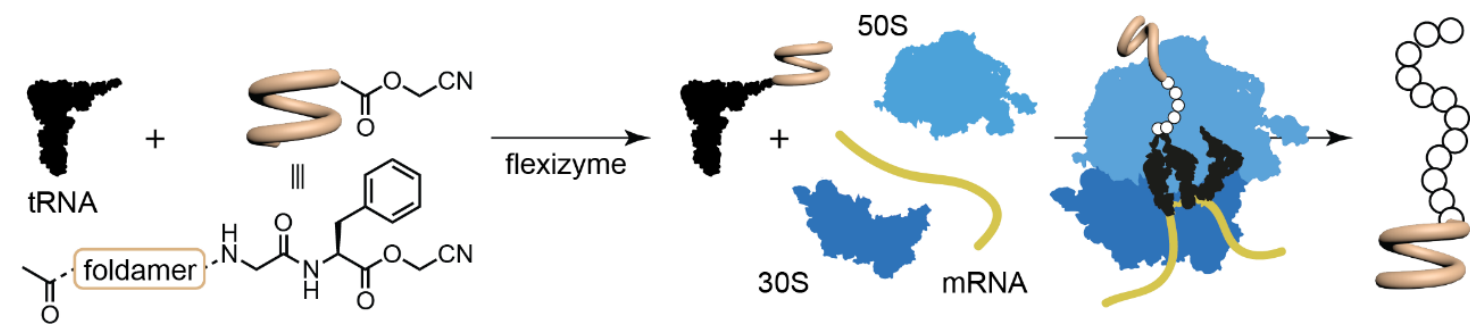

e

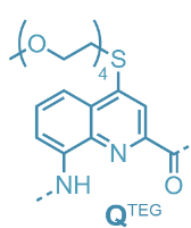

1: $A c-Q^{A s p}-G l y-P h e-C M E$

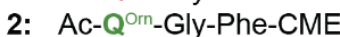

3: Ac- $Q^{\mathrm{TEG}}-\mathrm{Gly}-\mathrm{Phe}-\mathrm{CME}$

4: Ac-QQ-Gly-Phe-CME

5: Ac-QQQ-Gly-Phe-CME

\begin{abstract}
6: Ac-PQ-Gly-Phe-CME 7: Ac-QPQ-Gly-Phe-CME 8: Ac-PQPQ-Gly-Phe-CME 9: Ac-QPQPQ-Gly-Phe-CME 10: Ac-PPQPQ-Gly-Phe-CME 11: CIAc-PQPQ-Gly-Phe-CME
\end{abstract}

f

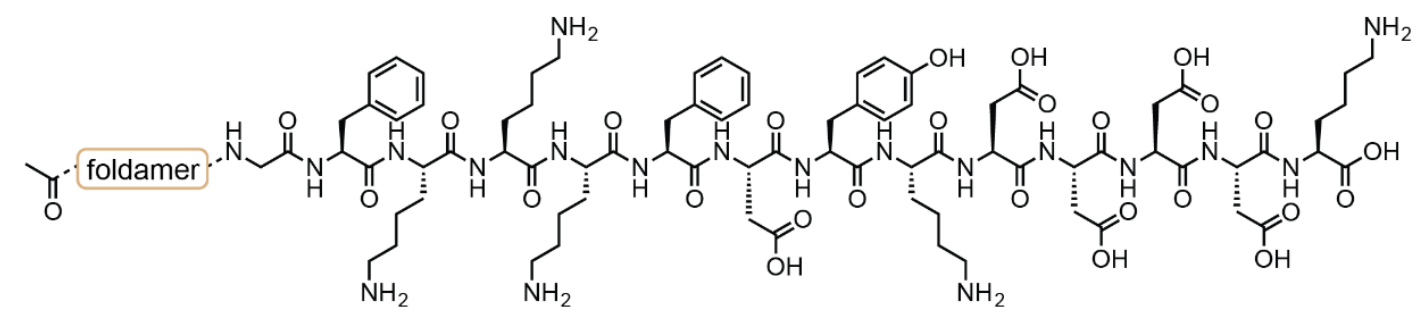

Figure 1 I Aromatic oligoamide foldamers and their initiation of in vitro translation. a, Quinolinebased ( $\mathbf{Q}$, centre) and pyridine-based ( $\mathbf{P}$, right) monomers with a comparable backbone (shown in bold) to that of a dipeptide (e.g. Gly-Gly, left). $\mathbf{Q}$ can be functionalized with a variety of side-chains $(R)$. b, Intramolecular $\mathrm{H}$-bonds (dotted lines, left) within $\mathbf{Q}_{\mathbf{n}}$ oligomers, together with $\square \square$ stacking, allows for the formation of stable helices as shown by the crystal structure of $\mathbf{Q}_{10}$ (right, side-chains have been omitted for clarity) ${ }^{32}$. c, Schematic representation of the acylation of tRNAs with $\mathrm{N}$-acetylated foldamers having a C-terminal glycyl-phenylalanyl cyanomethyl ester, by a flexizyme. Foldamer-tRNA adducts can be used to initiate in vitro translation to form foldamer-peptide hybrids (mRNA: yellow, ribosome 50S: light blue, ribosome 30S: dark blue, amino acids: white circles). d, Monomeric units of $\mathbf{Q}$ with aspartate-like (red), ornithine-like (green) and tetraethylene glycol (blue) side-chains, respectively. e, Foldamer substrates 1-11 used in this study (CME stands for cyanomethyl ester). $\mathbf{f}$, Structure of a foldamerpeptide (GFKKKFDYKDDDDK) hybrid. The C-terminal peptide segment DYKDDDDK is a flag-tag sequence. Unless stated otherwise, DNA/mRNA encoding of this peptide sequence was used.

Acylation of tRNA with foldamer substrates. Foldamer substrates were designed for attachment to tRNA, catalysed by the flexizyme 'eFx'. To ensure good yields, all substrates in this study included a C-terminal glycine spacer followed by a phenylalanine cyanomethylester (Gly-Phe-CME, Fig. 1c,e), which is an appropriate peptide-donor for tRNA acylation mediated by $\mathrm{eFx}^{5,33}$. Three substrates were synthesized containing $\mathbf{Q}$ monomers with a range of sidechains: aspartate-like (1), ornithine-like (2) and tetraethylene glycol (3) (Fig. 1d,e). Aminoacylation of these substrates was tested by charging onto an RNA microhelix, using an 
appropriate DMSO-containing reaction buffer (Supplementary Fig. 1). Note that this RNA microhelix was used as a tRNA mimic for the virtue of detection simplicity, as its acylation can be readily quantified using band intensity on acid polyacrylamide gel electrophoresis (PAGE) and as it has been established that the yield of microhelix charging correlates with the activity using tRNA ${ }^{5}$. Longer foldamers 4-11 comprised of several $\mathbf{Q}$ units with aspartate-like side chains and of $\mathbf{P}$ units were also synthesized and could be charged onto the RNA microhelix (Supplementary Figs 2-4), showing the high substrate tolerance of eFx. Since peptide synthesis in $E$. coli is initiated with $N$-formyl methionine-charged tRNA ${ }^{\text {fMet }}$ CAU, we have carried out the in vitro transcript acylation of tRNA ${ }^{\mathrm{fMet}} \mathrm{CAU}$ by foldamer substrates 1-11 and examined their use in translation.

Scope of in vitro translation initiation with appended $\mathbf{Q}_{n}$ oligomers. Initial experiments showed that foldamer-tRNA ${ }^{\mathrm{fMet}} \mathrm{CAU}$ from substrates $\mathbf{1 - 3}$, containing $\mathbf{Q}$ monomers with variable side-chains, could all initiate the synthesis of dodecapeptide KKKFDYKDDDDK (Fig. 1f). The expected foldamer-peptide hybrids were detectable by MALDI-TOF-MS (Fig. 2a,b and Supplementary Fig. 1). The efficiency of translation for these substrates were 10-20\% that of formyl methionine-initiated translation (Supplementary Fig. 5). These yields are good considering that the substrates are substantially larger and more rigid than formyl methionine; they compare in size with the largest appendages previously used in peptide synthesis initiation $8,15,16,18,34,35$. Furthermore, initiation appears to work regardless of the side-chain, and even a large tetraethylene glycol was accepted. Foldamer-tRNA ${ }^{\mathrm{fMet}} \mathrm{CAU}$ containing two or three $\mathbf{Q}$ units with aspartate-like side-chains were then tested in translation (from $\mathbf{4}$ and $\mathbf{5}$, respectively) (Fig. 2a). Initiation of peptide synthesis was possible using 4, albeit with a lower yield than using 1 (Supplementary Fig. 5), but no foldamer-peptide hybrid could be detected using 5 (Fig. 2a,b). To test whether delivery to the ribosome was preventing initiation with $\mathbf{5}$, we increased the concentration of initiation factor 2 (IF2), a key protein in this process, from 0.4 to $8 \mu \mathrm{M}$, but the foldamer-peptide hybrid was not observed. $\mathbf{Q}_{\mathbf{n}}$ oligomers adopt compact helical structures ${ }^{36}$ whose stability increases with oligomer length ${ }^{19,37}$. In these helices, five $\mathbf{Q}$ units span two helix turns. It is notable that foldamer $\mathbf{4}(\mathbf{Q Q}, 0.8$ turns), and not $\mathbf{5}(\mathbf{Q Q Q}, 1.2$ turns) is acceptable to the ribosome - only the latter can form a full turn of helix. We also recognise that the narrowest point in the ribosome exit tunnel is $\sim 1 \mathrm{~nm}$ in diameter ${ }^{38}$, similar to the diameter of a folded $\mathbf{Q}_{\mathbf{n}}$ helix without side-chains (Supplementary Fig. 6). It is therefore plausible that the helix of $\mathbf{5}$, will not pass this constriction if it cannot unfold. 
a

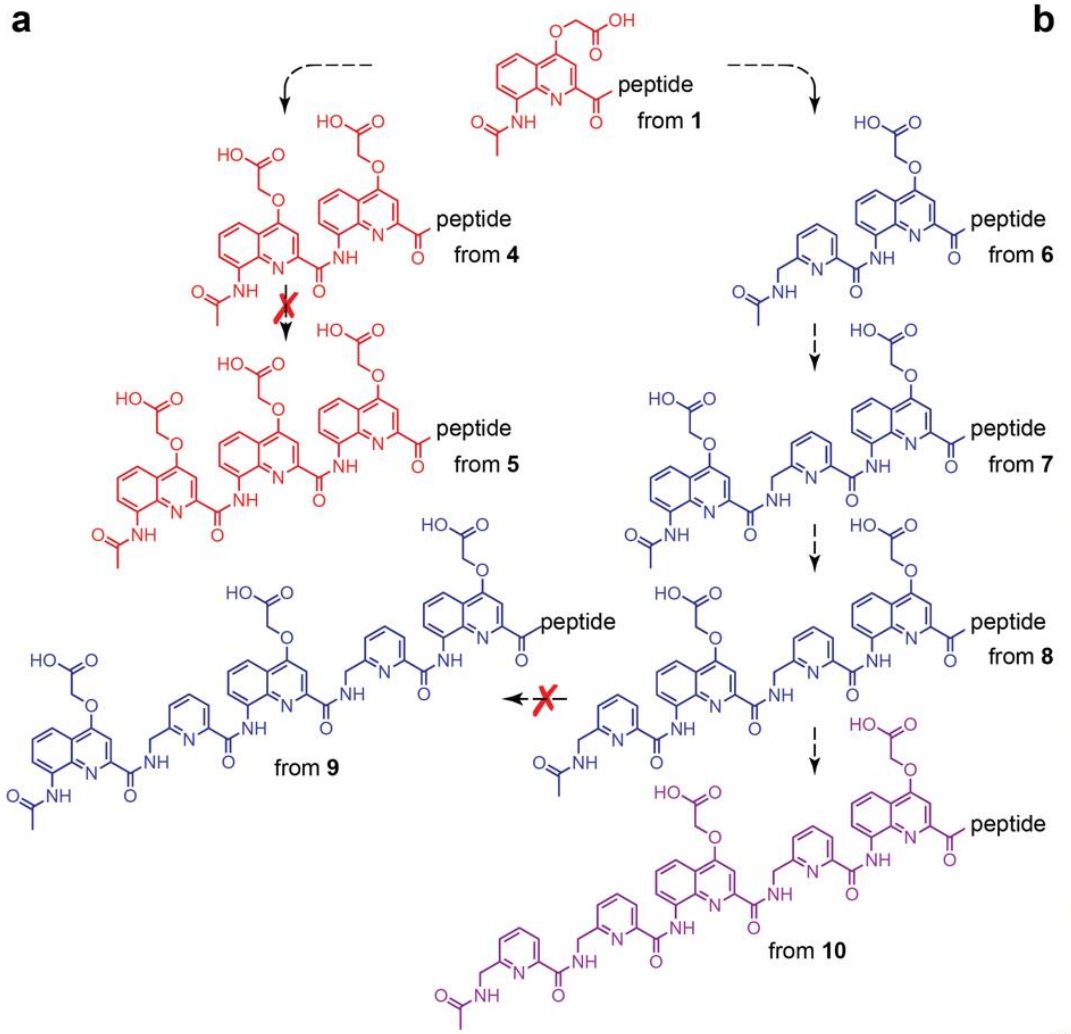

Ac-Q-peptide
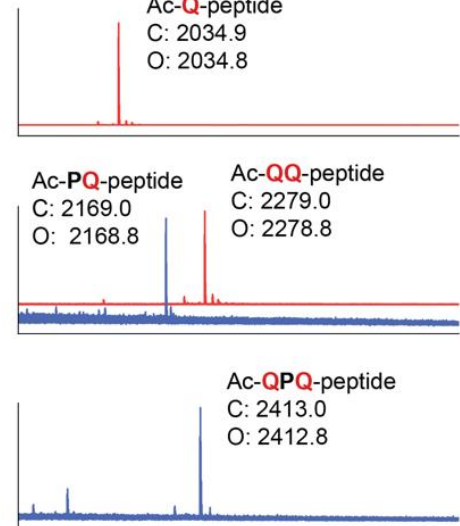

Ac-PQPQ-peptide

O: 2546.9

C: 2547.1

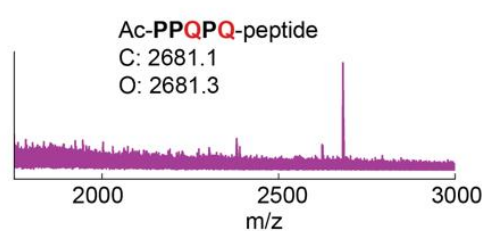

C

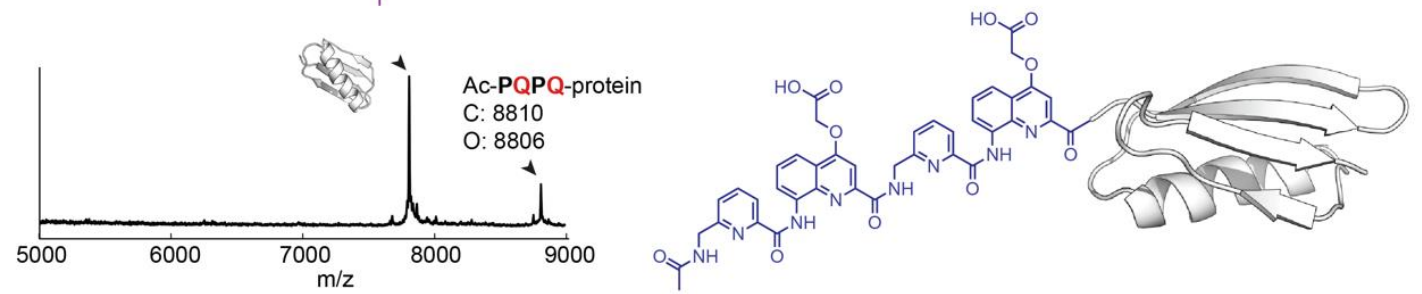

Figure 2 I In vitro translation of oligomeric foldamer-peptide/protein hybrids. a, Formulae of the expected targets (using substrates 1 and 4-10). 'peptide' refers to GFKKKFDYKDDDDK (shown in Fig. 1f). Dashed arrows represent the iterative exploration of foldamer sequences in this study. Red crosses indicate that the subsequent sequence was not translated (i.e. from 5 and 9). Thus, increasing the number of $\mathbf{Q}$ from 2 to 3 resulted in unsuccessful translation. Elongating $(\mathbf{P Q})_{2}$ by one $\mathbf{Q}$ also prevented translation. However, increasing the $\mathbf{P}$ content (using substrate 10) allowed a pentameric foldamerpeptide hybrid to be formed. b, MALDI-TOF-MS spectra of foldamer-peptide products successfully formed using in vitro translation. From top to bottom: peptide initiated with 1 (red); initiation using 4 (red) and 6 (blue); 7 (blue); 8 (blue); and 10 (purple). No product could be observed for substrates 5 and 9. c, MALDI-TOF-MS spectra (left) of a foldamer-protein hybrid formed using a DNA template for protein $\mathrm{G}$ (white, right). The major product corresponds to protein synthesis starting from the second (non$A \cup G$ ) codon. (C: calculated for $[\mathrm{M}+\mathrm{H}]^{+}, \mathrm{O}$ : observed mass).

Inclusion of pyridine-based monomers in $\mathbf{Q}_{\mathbf{n}}$ sequences. We sought to reduce the conformational stability of the foldamer helix to test whether it would permit translation of larger initiation units. This can be achieved by replacing $\mathbf{Q}$ with the more flexible 6-aminomethylpyridine $\mathbf{P}$ monomer (Fig. 1a). $\mathbf{P}$ has the same backbone atoms as $\mathbf{Q}$, and sequences combining $\mathbf{P}$ and $\mathbf{Q}$ fold into similarly shaped helices ${ }^{39,40}$. However, $\mathbf{P}$ possesses a smaller 
surface for aromatic stacking and an additional rotatable bond at the methylene group that interrupts conjugation; thus, both factors should contribute to lower the overwhelming stability of $\mathbf{Q}_{\mathbf{n}}$ helices. For instance, $\mathbf{P}_{\mathbf{n}}$ oligomers do not fold ${ }^{40}$. In sequences $\mathbf{6}-\mathbf{9}, \mathbf{P}$ and $\mathbf{Q}$ units alternate (Fig. 1e). Using tRNA ${ }^{\mathrm{fMet}} \mathrm{CAU}$ acylated with these substrates, translation could be initiated with 7 and 8 (albeit with lower efficiency than with 1-3) but not with the longest sequence 9 (Fig. 2a,b). As for 5 , increasing the concentration of IF2 from 0.4 to $8 \mu \mathrm{M}$ brought no improvement. To find a foldamer with five aryl units suitable for translation, we produced a variant of $\mathbf{9}$ having an $\mathbf{N}$-terminal $\mathbf{P}$ instead of $\mathbf{Q}$ (substrate 10). The foldamer-tRNA ${ }^{\mathrm{fMet}} \mathrm{CAU}$ charged with 10 was accepted by the ribosome and could be used in translation initiation.

Substrate $\mathbf{1 0}$ is long enough to span two full aromatic helix turns and amounts to a dodecameric peptide in size. It constitutes the largest appendage to tRNA ${ }^{\mathrm{fMet}} \mathrm{CAu}$ and largest single block delivered to the ribosome than has been used to date. Translation with $\mathbf{8}$ and $\mathbf{1 0}$, and not with $\mathbf{5}$ and $\mathbf{9}$, may result from the smaller size of $\mathbf{P}$ units, which lack a side chain and a main chain aromatic ring. Alternatively, their higher flexibility might allow transient unfolding when required during translation, e.g. to pass through the narrow constriction in the exit tunnel of the ribosome. However, one may also question whether the foldamer appendages have actually passed through the entire ribosome tunnel. The GFKKKFDYKDDDDK sequence formed in the above translation experiments is too short for the foldamer segment to protrude from the ribosomal exit tunnel before peptide synthesis is complete. Indeed, this would require 30-40 amino acids ${ }^{41,42}$. It is therefore possible that the foldamer-peptide hybrids are not fully traversing the exit tunnel, but are released after disassembly of the ribosome machinery.

To investigate the ability of these foldamers to navigate the exit tunnel, we used a longer mRNA template coding for Protein G (74 amino acids, including a C-terminal flag-tag) and $\mathrm{TRNA}_{\mathrm{fMet}}^{\mathrm{fAU}}$ loaded with substrates 6, 8 and 10. Foldamer-protein hybrids could be synthesized by the ribosome and correct product observed using MALDI-TOF-MS after translation (Fig. 2c and Supplementary Fig. 7). This observation clearly indicates that these foldamers are indeed passing entirely through the exit tunnel. It remains to be determined whether this reflects a broad tolerance of the ribosome tunnel for a range of non-peptidic substrates or whether aromatic foldamers happen to be a favourable case. Noteworthy is the concomitant presence of translated products lacking the foldamer initiators (Supplementary Fig. 7). It is possible that, after assembly of the ribosome-mRNA complex, dissociation of the large foldamer-tRNA ${ }^{\mathrm{fMet}} \mathrm{CA \cup}$ occurs followed by the restart of translation at the second codon.

Solution studies of foldamer helices. To underpin our hypothesis on unfolding of foldamers in the ribosome exit tunnel, we investigated their conformation and stability in an aqueous environment. Foldamers 12-15 were synthesized, which correspond to initiators 5, 7, 8 and 9 lacking the C-terminal Phe-CME, respectively. Multidimensional Nuclear Magnetic Resonance (NMR) allowed for the complete assignment of their ${ }^{1} \mathrm{H}$ NMR spectra (Supplementary Figs 10- 
13). Rotating-frame Overhauser spectroscopy (ROESY) experiments showed correlations indicating that all are helically folded (Supplementary Figs 14-17). Although the patterns and intensities of NOE signals were not entirely identical, correlations between amide protons of adjacent residues were identified in all cases (Fig. 3a), consistent with the canonical quinoline helix in which amide protons reside at the inner rim (Fig. 3b ${ }^{40,43}$. Other medium-range NOE signals between nonadjacent residues ( $i$ to $i+2$ or $i$ to $i+3)$ represent typical through-space correlations in quinoline helices where side chain, aromatic, and Gly protons locate on the outer rim (Fig. 3b) ${ }^{31,43}$. The ${ }^{1} \mathrm{H}$ NMR spectra of 12-15 all featured at least one strongly upfield shifted singlet near 6.4 ppm (Fig. 3e), corresponding to the $\mathrm{H} 3$ proton of the first aromatic ring starting from the $\mathbf{C}$-terminus (always a $\mathbf{Q}$ ) that is shielded by the ring current effect of the stacked C-terminal quinoline in the helical conformation ${ }^{19}$.

a

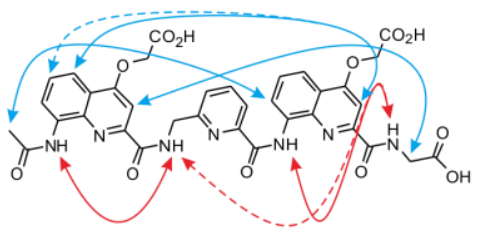

12: Ac-QPQ-Gly-OH

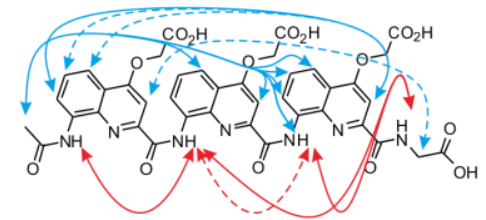

13: Ac-QQQ-Gly-OH
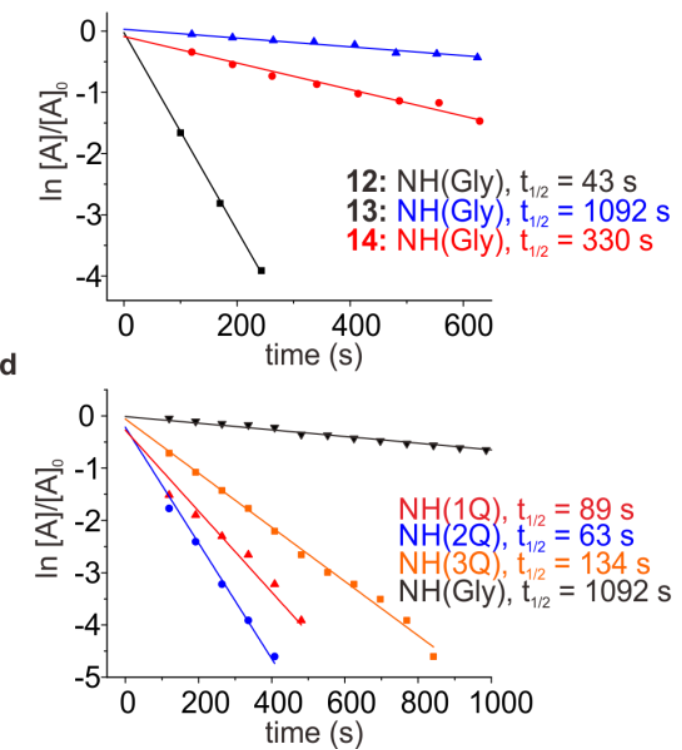

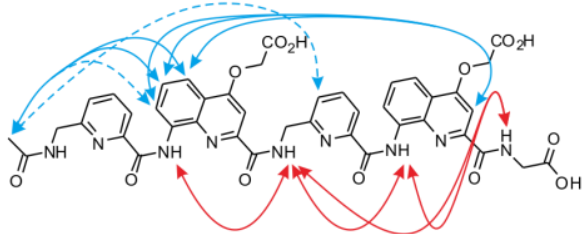

14: Ac-PQPQ-Gly-OH

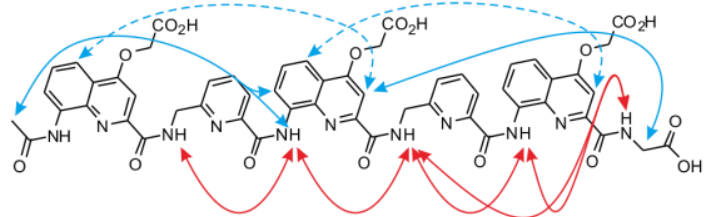

15: Ac-QPQPQ-Gly-OH

e

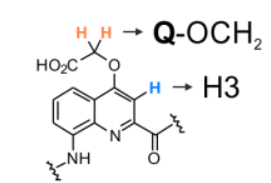

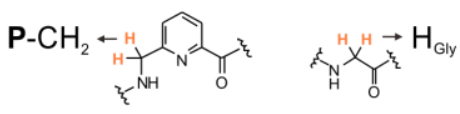
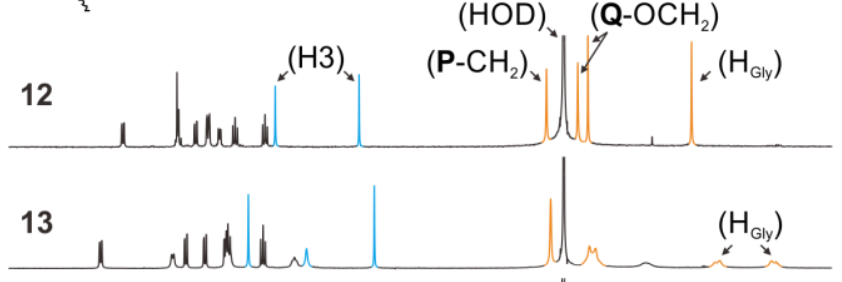

14

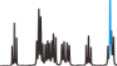

15

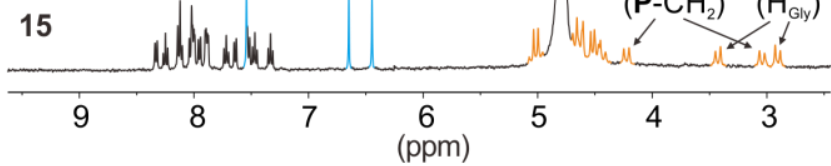

Figure 3 I NMR studies of aromatic foldamer segments. a, Chemical formulae of aromatic foldamer segments used for solution-state conformation studies with identified NOEs in aqueous solution (9:1 $\mathrm{H}_{2} \mathrm{O} / \mathrm{D}_{2} \mathrm{O}, 50 \mathrm{mM} \mathrm{NaHCO}_{3}$ ). Red arrows indicate correlations between backbone amide protons. Blue arrows indicate correlations between sequentially nonadjacent residues. Solid and dashed arrows 
indicate strong and medium/weak interactions, respectively. b, Molecular model of 12 with observed NOEs. c-d, Pseudo first-order rate plots of $\mathrm{NH} / \mathrm{ND}$ exchange and half-life for $\mathrm{NH}(\mathrm{Gly})$ in $\mathrm{D}_{2} \mathrm{O}$ at $298 \mathrm{~K}$ of 12, 13, and 14 (c), and amide protons of 13 (d). Monitoring of $\mathrm{NH}(\mathrm{Gly})$ of 15 was not possible due to signal overlap. e, Parts of ${ }^{1} \mathrm{H}$ NMR spectra of 12, 13, 14, and 15 in $\mathrm{D}_{2} \mathrm{O}$ with $50 \mathrm{mM} \mathrm{NaHCO}_{3}$ at $298 \mathrm{~K}$. Peaks marked with blue colour indicate $\mathrm{H} 3$ protons of quinoline rings. Peaks marked with orange colour indicate $\mathrm{CH}_{2}$ protons of $\mathbf{Q}$ (side chain), $\mathbf{P}$ (main chain) and $\mathrm{Gly}$ (main chain) residues.

Having established folding, we next investigated helix stability. NH/ND exchange rates allow for the assessment of exposure to the surrounding water and in part reflect conformational dynamics (Supplementary Figs 18-21). Pseudo-first order rate constants of amide NH/ND exchange in 12-15 could be calculated and were shown to be significantly lower for 13 and 15. Within a given molecule, NH/ND exchange rates vary according to chemical nature and position in the sequence (Fig. 3d). When examining comparable functions, e.g. the terminal $\mathrm{NH}$ (Gly) of different oligomers, exchange was found to be faster for $\mathbf{1 2}$ and $\mathbf{1 4}$ than for 13 (Fig. 3c). The range of rates is considerable: amide protons of 12 except its $\mathrm{NH}$ (Gly) are quantitatively exchanged within $100 \mathrm{~s}$ whereas the $\mathrm{NH}$ of the central $\mathbf{Q}$ of pentamer 15 exchanges with a half-life of $66 \mathrm{~min}$ (Supplementary Figs 18 and 21). Overall, stability increases upon increasing length and upon increasing the proportion of $\mathbf{Q}$ and may well be the factor that prevents translation initiation with $\mathbf{5}$ and $\mathbf{9 .}$

Conformational stability was also reflected in ${ }^{1} \mathrm{H}$ NMR spectra. Helical conformations are inherently chiral even if the molecules possess no stereogenic centres. As a result, $\mathrm{CH}_{2}$ protons in $\mathbf{Q}$ (side chain) and $\mathbf{P}$ and Gly (main chain) are diastereotopic and their signals may be anisochronous when exchange between left- and right-handed helices is slow on NMR time scale ${ }^{19}$, i.e. when the helices have sufficient stability. Consistent with the higher stability of 13 and 15 revealed by $H / D$ exchange rates, anisochronous $\mathrm{CH}_{2}$ signals are obvious in the spectra of these sequences, where multiple $A B$ systems are present (Fig. $3 e$ ) that coalesced at elevated temperature $\left(45^{\circ} \mathrm{C}\right.$, Supplementary Figs 23 and 25). In contrast, the diastereotopic protons appear as sharp averaged singlets in the spectrum of $\mathbf{1 2}$ as left- and right-handed helices exchange rapidly (Supplementary Fig. 22). The spectrum of 14 shows an intermediate situation with some broadening (Supplementary Fig. 24) suggesting that its dynamics, albeit faster than for $\mathbf{1 3}$ and 15, are slower than for $\alpha$-peptidic chains for which fast exchange on the NMR timescale is observed ${ }^{44}$. In summary, these results suggest that translation initiation occurs only when appended foldamer helices have a sufficient ability to transiently unfold to pass through the ribosome exit tunnel, and that this is allowed for helices that are nevertheless more stable than a typical, isolated peptide helix. 
Ribosomal synthesis of macrocyclic foldamer-peptide hybrids. To further expand the chemical diversity produced by the ribosome and to exploit ribosomal uptake of helical foldamers with better defined conformations than short peptides, we endeavoured to express foldamer-peptide cyclic hybrids. In a macrocycle, the peptide and foldamer are attached at two points which might result in a coupling of their respective conformations. Genetic code reprogramming with an N-terminal chloroacetyl (CIAc) amino acid allows for efficient macrocyclization through thioether formation with the thiol of a cysteine residue further along the sequence ${ }^{12}$. Precursor 11 (Fig. 1e) was prepared to include a terminal CIAc to form foldamer-containing macrocycles (Fig. 4a), and this substrate could initiate translation (Supplementary Fig. 4). Additional mRNA templates were designed to encode a cysteine either immediately after the foldamer initiator (template C2) or further along the peptide (templates C3, C4, C5) and these were used for in vitro translation, initiated using 11. Within 30 minutes of translation, cyclized species were detected as the major product for templates C4 and C5, whereas linear foldamer-peptide hybrids were still the major products for C2 and C3 (Fig. 4a). After 10 mins translation, and release from the ribosome using EDTA, cyclization for C2 and C3 proceeded at a slower, similar rate $\left(k_{\mathrm{cyc}}=3 \times 10^{-4} \mathrm{~s}^{-1}\right.$, Supplementary Figs 8,9$)$.

The effect of peptide stapling by a foldamer on the macrocycle conformation was then investigated in solution. Macrocycles 16 and 17 were synthesized as analogues of cyclized translation products from C4 and C5 templates (Fig. 4b). Their circular dichroism (CD) spectra showed positive bands at 280 and $340 \mathrm{~nm}$, indicating bias toward a right-handed foldamer helix ${ }^{45}$ as a consequence of the L-chirality of the peptide. Heating had little effect on the CD spectra (Fig. 4c and Supplementary Figs 26,27), hinting at helix stability even under harsh conditions such as 7:3 $\mathrm{H}_{2} \mathrm{O} / \mathrm{DMSO}$ (vol/vol) at $338 \mathrm{~K} .{ }^{1} \mathrm{H}$ NMR spectra of 16 and 17 showed poorly resolved signals at room temperature (Supplementary Figs 28,29), presumably due to nonspecific aggregation promoted by their amphipathic and zwitterionic nature. However, sharp spectra at $338 \mathrm{~K}$ (Fig. 4d) allowed for the observation of quinoline $\mathrm{H} 3$ protons singlets near 6.4 ppm, indicating shielding by aromatic stacking. In addition, $\mathrm{NH}-\mathrm{CH}_{2}{ }^{\beta}$-couplings of $\mathbf{P}$ units showed obvious diastereotopic patterns (Fig. 4e and Supplementary Figs 30,31). Thus, the PQPQ segment in both 16 and $\mathbf{1 7}$ is helical, chirally biased, and stabilized with respect to noncyclic sequence 14. Helix conformation control by stereocentres can be remote ${ }^{46}$. Here the nearest stereogenic centres are five atoms away from the foldamer helix at both $\mathrm{C}$ and $\mathrm{N}$ termini, which much contrasts with earlier examples of absolute handedness control ${ }^{45}$. 

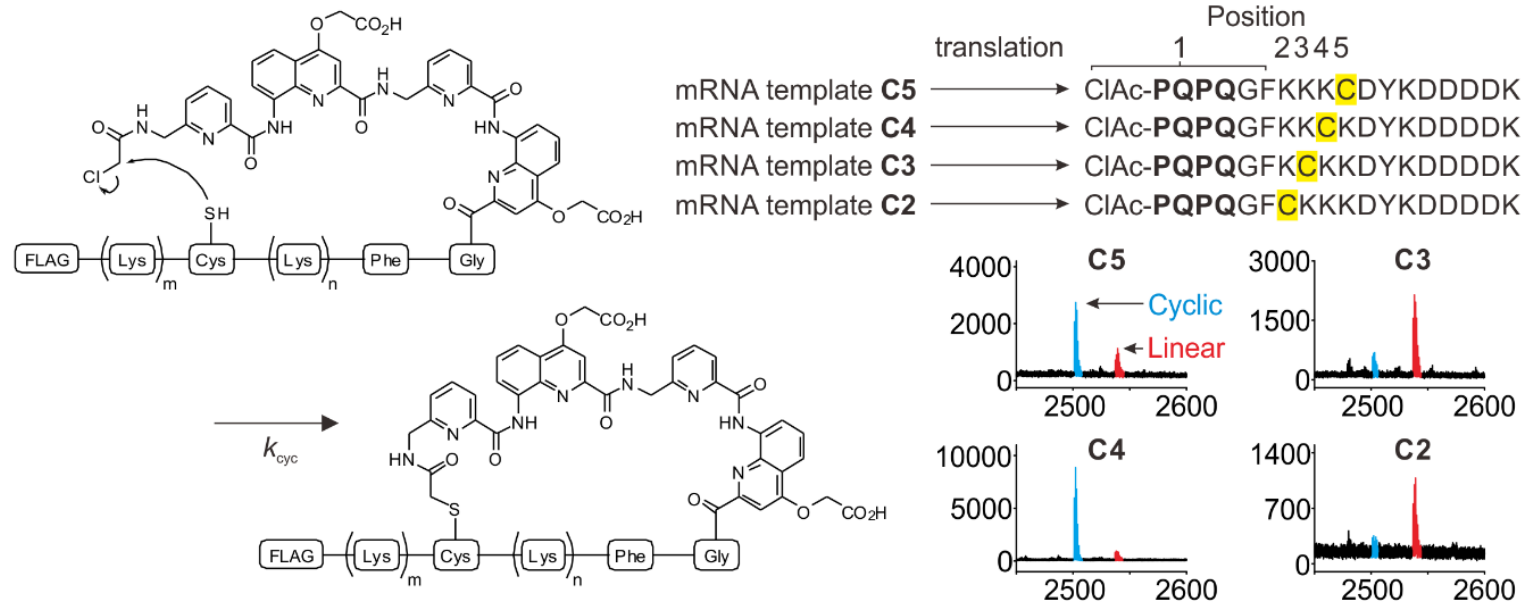

b

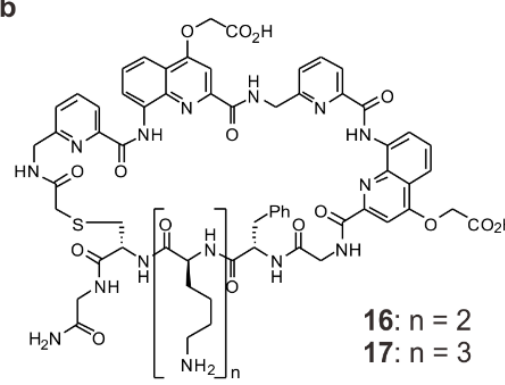

c

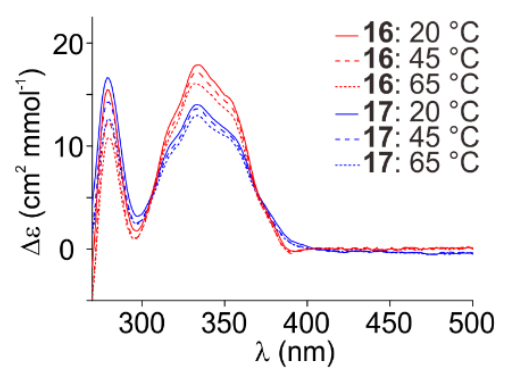

d

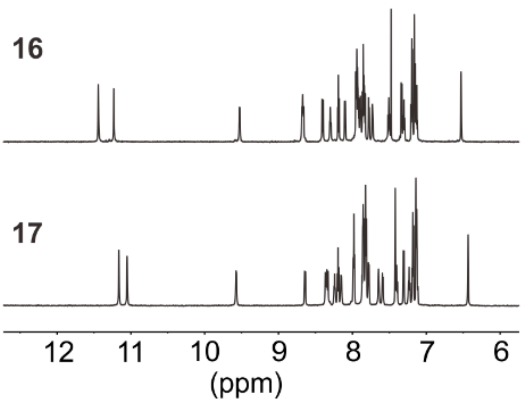

e 16

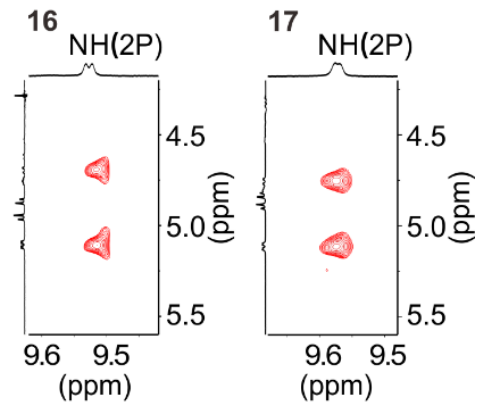

f

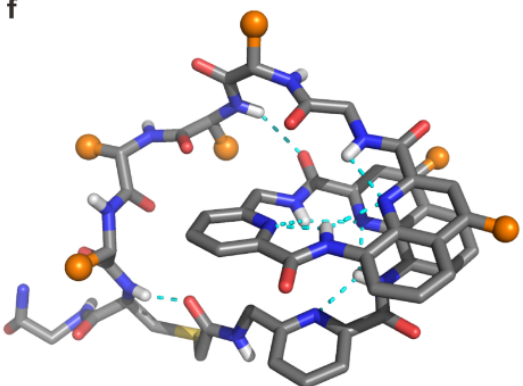

g

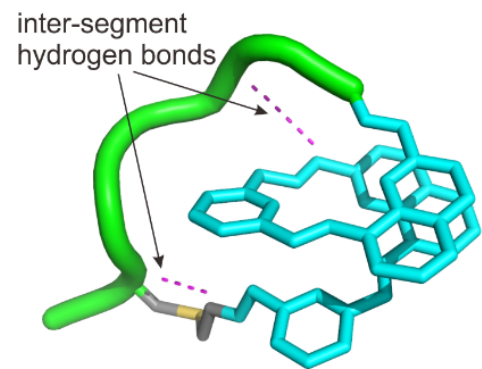

Figure 4 I Macrocyclization of foldamer-peptide hybrids. a, Macrocyclization by thioether formation from an N-terminal chloroacetyl group and a cysteine thiol, and corresponding MALDI-TOF-MS spectra. b. Synthesized foldamer-peptide macrocycles. c, Circular dichroism spectra of 16 and 17 in $\mathrm{H}_{2} \mathrm{O} / \mathrm{DMSO}-d_{6}(7: 3, \mathrm{vol} / \mathrm{vol})$ at variable temperatures. $\mathbf{d}$, Selected parts of ${ }^{1} \mathrm{H}$ NMR spectra of 16 and 17 in $\mathrm{H}_{2} \mathrm{O} / \mathrm{DMSO}-d_{6}(7: 3, \mathrm{vol} / \mathrm{vol})$ at $338 \mathrm{~K}$. e, Selected parts of TOCSY spectra of 16 and 17 in $\mathrm{H}_{2} \mathrm{O} / \mathrm{DMSO}-d_{6}(7: 3, \mathrm{vol} / \mathrm{vol})$ at $338 \mathrm{~K}$ showing $\mathrm{NH}-\mathrm{CH}_{2} \mathrm{~J}$-couplings. f, Crystal structure of compound 17. Side-chains of lysine, phenylalanine and $\mathbf{Q}$ are shown as orange spheres. $\mathbf{g}$, Stick-loop representation of the crystal structure of $\mathbf{1 7}$. The peptide backbone shown as a green loop is held by the helical aromatic foldamer shown as a cyan stick. The thioether linkage is shown in yellow.

Crystals of $\mathbf{1 7}$ could be obtained but diffracted only at $2.5 \AA$. The D- enantiomer of 17 was then also synthesized and mixed with $\mathrm{L}-\mathbf{1 7}$ to produce a racemate. Crystals of racemic 17 diffracted at $0.9 \AA$ and the structure in the solid state could be resolved in centrosymmetrical space group $\mathrm{P} \overline{1}$ (Supplementary Figs 32,33). Two independent molecules in the asymmetric unit show essentially superimposable main chain conformation, suggesting that this conformation is robust (Supplementary Fig. 34). The structure proved to be consistent with 
solution phase observations (Fig. 4f,g and Supplementary Movie 1). The aromatic segment PQPQ of L-17 is folded into a compact right-handed helix. Conversely, D-17 is left-handed. The helical fold is similar to that of canonical $\mathbf{Q}_{n}$ helices. In contrast, the six-amino-acid peptide segment adopts a loop-like extended conformation. Two hydrogen bonds are established between two peptidic $\mathrm{NH}$ protons belonging to Lys2 and Cys5, and the two $\mathrm{CO}$ of the carbonyamino-methyl groups in position 6 of the two $\mathbf{P}$ units. These inter-segment interactions presumably mediate helix handedness control while restraining the peptide conformation. The peptide side-chains are not involved, suggesting that the conformation may be sequence independent. The reciprocal conformational effects between these chemically distinct biotic and abiotic folded entities - a peptide and an aromatic oligoamide - is quite unique. The structure mirrors stapled helical peptides ${ }^{22}$ in which the peptide is $\alpha$-helical and the hydrocarbon staple is extended. Similar structures have also been observed in thioetherbridged macrocyclic peptides ${ }^{47,48}$. Such macrocycles were synthesized by the ribosome and were selected from large libraries to bind to a particular partner protein. When bound, these macrocycles use a helix to present a peptide loop for binding, with the helix involved to different degrees in the binding interface (Supplementary Fig. 35). This raises an attractive possibility of using the structures described here, helical foldamers presenting a peptide loop, for molecular recognition.

\section{Conclusions}

We have demonstrated that aromatic foldamer-appended peptides could be synthesized by the ribosome using genetic code reprogramming. Foldamer-tRNA prepared by flexizyme can be delivered as a single unit to the ribosome and successfully initiated peptide synthesis. The foldamers represent not only the largest single building blocks to be accepted by the ribosome, but also chemical entities very distinct from the peptide sequences that the ribosome has been optimized to accept by evolution. We find that translation requires a certain degree of conformational flexibility, possibly to allow for the unfolding of the foldamer segments to pass through ribosome exit tunnel. Yet, foldamers with folding propensities stronger than that of peptides of comparable sizes - a noncyclic decapeptide rarely folds - are accepted for translation. It can be conceived that fine tuning of folding-unfolding behaviour might allow translation of even larger appendages. In addition, macrocyclization chemistry was successfully applied to these foldamer-peptide hybrids. Helically folded aromatic foldamers in cyclic peptides act as a novel class of peptide linker that can serve as a structural scaffold to present the peptide in 3-dimensional space. Solution and solid state studies reveal reciprocal influence of the peptide and foldamer helix conformations, presumably by mutual reduction of the entropy penalty inherent to folding. For this type of hybrid molecule, one may expect functional improvements for the peptide regions in terms of selectivity and binding affinity to 
target biomolecules. Conversely, the foldamer may be highly water soluble and possess cell penetrating properties. ${ }^{28}$ An obvious subsequent step is to investigate the amenability of these constructs to sequence randomization and selection of functional species from libraries.

Data availability. The crystallographic data and experimental details of the structural refinement for the X-ray crystal structure of $\mathbf{1 7}$ have been deposited at the Cambridge Crystallographic Data Centre, under deposition numbers CCDC 1554263. These data can be obtained free of charge from the Cambridge Crystallographic Data Centre (http://www.ccdc.cam.ac.uk/data_request/cif). Other data that support the findings of this study (spectroscopic or mass spectrometric data) are available from the corresponding author on reasonable request.

Acknowledgments. This work was mainly supported by a joint ANR-JST grant (ANR-14JTIC-2014-003 and JST-SICORP) to H.S. and I.H. It was partly supported by European Research Council under the European Union's Seventh Framework Programme (ERC-2012AdG-320892) to I.H., a Japan Society for the Promotion of Science post-doctoral fellowship (P13766) to J.M.R., and Basic Science Research Program through the National Research Foundation of Korea (NRF) funded by the Ministry of Education (2016R1A6A3A03006364) to SK. This work has benefited from the facilities and expertise of the Biophysical and Structural Chemistry platform at IECB, CNRS UMS3033, INSERM US001, Bordeaux University, France.

Author Contributions. S.K. and J.M.R. contributed equally to this work. S.K. and S.J.D. synthesized new compounds. S.K. carried out solution conformational studies. J.M.R. performed in vitro aminoacylation and translation experiments. P.K.M. carried out crystallographic studies. All authors contributed to designing the research, to discussing the results and to writing the manuscript.

Additional Information. Supplementary Information is linked to the online version of the paper at http://www.nature.com/nature. Reprints and permissions information is available at http://www.nature.com/reprints. Correspondence and requests for materials should be addressed to H.S (hsuga@chem.s.u-tokyo.ac.jp) or to I.H. (ivan.huc@cup.Imu.de).

Competing financial interests. The authors declare no competing financial interests. 


\section{References}

1 Fischer, N. et al. Structure of the E. coli ribosome-EF-Tu complex at $<3$ A resolution by Cscorrected cryo-EM. Nature 520, 567-570, (2015).

2 Ernst, R. J. et al. Genetic code expansion in the mouse brain. Nat. Chem. Biol. 12, 776-778, (2016).

3 Lajoie, M. J. et al. Genomically recoded organisms expand biological functions. Science 342, 357-360, (2013).

4 Maini, R. et al. Ribosome-Mediated Incorporation of Dipeptides and Dipeptide Analogues into Proteins in Vitro. J. Am. Chem. Soc. 137, 11206-11209, (2015).

5 Murakami, H., Ohta, A., Ashigai, H. \& Suga, H. A highly flexible tRNA acylation method for non-natural polypeptide synthesis. Nat. Methods 3, 357-359, (2006).

6 Hartman, M. C., Josephson, K., Lin, C. W. \& Szostak, J. W. An expanded set of amino acid analogs for the ribosomal translation of unnatural peptides. PLoS One 2, e972, (2007).

7 Shimizu, Y. et al. Cell-free translation reconstituted with purified components. Nat. Biotechnol. 19, 751-755, (2001).

8 Rogers, J. M. \& Suga, H. Discovering functional, non-proteinogenic amino acid containing, peptides using genetic code reprogramming. Org. Biomol. Chem. 13, 9353-9363, (2015).

9 Katoh, T., Tajima, K. \& Suga, H. Consecutive Elongation of D-Amino Acids in Translation. Cell Chem Biol 24, 46-54, (2017).

10 Fujino, T., Goto, Y., Suga, H. \& Murakami, H. Reevaluation of the D-amino acid compatibility with the elongation event in translation. J. Am. Chem. Soc. 135, 1830-1837, (2013).

11 Fujino, T., Goto, Y., Suga, H. \& Murakami, H. Ribosomal Synthesis of Peptides with Multiple beta-Amino Acids. J. Am. Chem. Soc. 138, 1962-1969, (2016).

12 Goto, Y. et al. Reprogramming the translation initiation for the synthesis of physiologically stable cyclic peptides. ACS Chem. Biol. 3, 120-129, (2008).

13 Ohta, A., Murakami, H., Higashimura, E. \& Suga, H. Synthesis of polyester by means of genetic code reprogramming. Chem. Biol. 14, 1315-1322, (2007).

14 Kawakami, T., Murakami, H. \& Suga, H. Ribosomal synthesis of polypeptoids and peptoidpeptide hybrids. J. Am. Chem. Soc. 130, 16861-16863, (2008).

15 Goto, Y. \& Suga, H. Translation initiation with initiator tRNA charged with exotic peptides. J. Am. Chem. Soc. 131, 5040-5041, (2009).

16 Torikai, K. \& Suga, H. Ribosomal synthesis of an amphotericin-B inspired macrocycle. J. Am. Chem. Soc. 136, 17359-17361, (2014).

17 Passioura, T. \& Suga, H. A RaPID way to discover nonstandard macrocyclic peptide modulators of drug targets. Chem. Commun. 53, 1931-1940, (2017).

18 Terasaka, N., Hayashi, G., Katoh, T. \& Suga, H. An orthogonal ribosome-tRNA pair via engineering of the peptidyl transferase center. Nat. Chem. Biol. 10, 555-557, (2014).

19 Jiang, H., Léger, J. M. \& Huc, I. Aromatic delta-peptides. J. Am. Chem. Soc. 125, 3448-3449, (2003).

20 Boersma, M. D. et al. Evaluation of diverse alpha/beta-backbone patterns for functional alpha-helix mimicry: analogues of the Bim BH3 domain. J. Am. Chem. Soc. 134, 315-323, (2012).

21 Fuller, A. A. et al. Evaluating beta-turn mimics as beta-sheet folding nucleators. Proc. Natl. Acad. Sci. U. S. A. 106, 11067-11072, (2009).

Walensky, L. D. \& Bird, G. H. Hydrocarbon-stapled peptides: principles, practice, and progress. J. Med. Chem. 57, 6275-6288, (2014).

23 Lao, B. B. et al. In vivo modulation of hypoxia-inducible signaling by topographical helix mimetics. Proc. Natl. Acad. Sci. U. S. A. 111, 7531-7536, (2014).

24 Fremaux, J. et al. alpha-Peptide-Oligourea Chimeras: Stabilization of Short alpha-Helices by Non-Peptide Helical Foldamers. Angew. Chem. Int. Ed. Engl. 54, 9816-9820, (2015).

25 Cheng, P. N. \& Nowick, J. S. Giant macrolactams based on beta-sheet peptides. J. Org. Chem. 76, 3166-3173, (2011).

26 Goodman, C. M., Choi, S., Shandler, S. \& DeGrado, W. F. Foldamers as versatile frameworks for the design and evolution of function. Nat. Chem. Biol. 3, 252-262, (2007).

27 Guichard, G. \& Huc, I. Synthetic foldamers. Chem. Commun. 47, 5933-5941, (2011).

28 Gillies, E. R., Deiss, F., Staedel, C., Schmitter, J. M. \& Huc, I. Development and biological assessment of fully water-soluble helical aromatic amide foldamers. Angew. Chem. Int. Ed. Engl. 46, 4081-4084, (2007).

29 Delaurière, L. et al. Deciphering aromatic oligoamide foldamer-DNA interactions. Angew. Chem. Int. Ed. Engl. 51, 473-477, (2012). 
Kudo, M., Maurizot, V., Kauffmann, B., Tanatani, A. \& Huc, I. Folding of a linear array of alpha-amino acids within a helical aromatic oligoamide frame. J. Am. Chem. Soc. 135, 96289631, (2013).

31 Hu, X., Dawson, S. J., Nagaoka, Y., Tanatani, A. \& Huc, I. Solid-Phase Synthesis of WaterSoluble Helically Folded Hybrid alpha-Amino Acid/Quinoline Oligoamides. J. Org. Chem. 81, 1137-1150, (2016).

32 Gillies, E. R., Dolain, C., Léger, J. M. \& Huc, I. Amphipathic helices from aromatic amino acid oligomers. J. Org. Chem. 71, 7931-7939, (2006).

33 Saito, H., Kourouklis, D. \& Suga, H. An in vitro evolved precursor tRNA with aminoacylation activity. Embo Journal 20, 1797-1806, (2001).

34 Goto, Y., Ashigai, H., Sako, Y., Murakami, H. \& Suga, H. Translation initiation by using various N-acylaminoacyl tRNAs. Nucleic Acids Symp. Ser., 293-294, (2006).

35 Goto, Y., Murakami, H. \& Suga, H. Initiating translation with D-amino acids. Rna 14, 13901398, (2008).

36 Qi, T. et al. Solvent dependence of helix stability in aromatic oligoamide foldamers. Chem. Commun. 48, 6337-6339, (2012).

37 Delsuc, N. et al. Kinetics of helix-handedness inversion: folding and unfolding in aromatic amide oligomers. Chemphyschem 9, 1882-1890, (2008).

38 Voss, N. R., Gerstein, M., Steitz, T. A. \& Moore, P. B. The geometry of the ribosomal polypeptide exit tunnel. J. Mol. Biol. 360, 893-906, (2006).

39 Sanchez-Garcia, D. et al. Nanosized hybrid oligoamide foldamers: aromatic templates for the folding of multiple aliphatic units. J. Am. Chem. Soc. 131, 8642-8648, (2009).

40 Baptiste, B., Douat-Casassus, C., Laxmi-Reddy, K., Godde, F. \& Huc, I. Solid phase synthesis of aromatic oligoamides: application to helical water-soluble foldamers. J. Org. Chem. 75, 7175-7185, (2010).

41 Cabrita, L. D. et al. A structural ensemble of a ribosome-nascent chain complex during cotranslational protein folding. Nat. Struct. Mol. Biol. 23, 278-285, (2016). Nilsson, O. B. et al. Cotranslational Protein Folding inside the Ribosome Exit Tunnel. Cell. Rep. 12, 1533-1540, (2015).

43 Dolain, C. et al. Solution structure of quinoline- and pyridine-derived oligoamide foldamers. Chemistry 11, 6135-6144, (2005).

44 Sola, J., Helliwell, M. \& Clayden, J. N-versus C-terminal control over the screw-sense preference of the configurationally achiral, conformationally helical peptide motif Aib(8)GlyAib(8). J. Am. Chem. Soc. 132, 4548-4549, (2010).

45 Kendhale, A. M. et al. Absolute control of helical handedness in quinoline oligoamides. J. Org. Chem. 76, 195-200, (2011).

$46 \mathrm{Li}, \mathrm{J}$. et al. An in-tether sulfoxide chiral center influences the biophysical properties of the $\mathrm{N}$ capped peptides. Bioorg Med Chem 25, 1756-1761, (2017).

47 Kodan, A. et al. Structural basis for gating mechanisms of a eukaryotic P-glycoprotein homolog. Proc. Natl. Acad. Sci. U. S. A. 111, 4049-4054, (2014).

$48 \mathrm{Yu}, \mathrm{H}$. et al. Macrocycle peptides delineate locked-open inhibition mechanism for microorganism phosphoglycerate mutases. Nat. Commun. 8, 14932, (2017). 